\title{
The plerion nebula in IC 443: The XMM-Newton view
}

\author{
F. Bocchino ${ }^{1,2}$ and A. M. Bykov ${ }^{3}$ \\ 1 Astrophysics Division, Space Science Dept. ESA-ESTEC, Postbus 299, 2200 AG Noordwijk, The Netherlands \\ 2 Osservatorio Astronomico di Palermo, Piazza del Parlamento 1, 90134 Palermo, Italy \\ 3 A. F. Ioffe Institute for Physics and Technology, 194021 St. Petersburg, Russia
}

Received 11 April 2001 / Accepted 15 June 2001

\begin{abstract}
XMM-Newton observations of the X-ray feature 1SAX J0617.1+2221 in the IC 443 supernova remnant are reported. We resolve the structure of the nebula into a compact core with a hard spectrum of photon index $\gamma=1.63_{-0.10}^{+0.11}$ in the $2-10 \mathrm{keV}$ energy range. The nebula also has an extended $\left(\sim 8^{\prime} \times 5^{\prime}\right) \mathrm{X}$-ray halo, much larger than the radio emission extension. The photon index softens, following a linear scaling with distance from the centroid, similar to other known X-ray plerions. The index range is compatible with synchrotron burn-off models. All the observational evidence points toward a confirmation of the plerionic nature of the nebula, as recently suggested by a Chandra observation, but with characteristics more similar to "non Crab-like" plerions. We discuss the implications on the synchrotron nebula magnetic field if the $>100 \mathrm{MeV}$ emission reported by CGRO EGRET is produced by the synchrotron emission. We also constrain the thermal emission of the central object, arguing that the surface temperature should be around $0.1 \mathrm{keV}$, although other possible fits cannot be excluded on the base of the XMM-Newton data.
\end{abstract}

Key words. pulsars: general - shock waves - ISM: supernova remnants - ISM: individual object: IC 443

\section{Introduction}

The processes of conversion of pulsar spin-down energy into high-energy emission in pulsar wind nebula are of great physical interest (e.g., Kennel \& Coroniti 1984; Chevalier 2000). Modern arcsec resolution instruments such as on XMM-Newton and Chandra that are sensitive to photons up to $10 \mathrm{keV}$ provide unique possibilities to study pulsar nebulae, because the non-thermal emission of the nebulae may be easily detected and studied in this band (e.g., Vela-X, Helfand et al. 2001; Pavlov et al. 2001; G21.5-0.9, Slane et al. 2000; Warwick et al. 2001; and 3C 58, Bocchino et al. 2001), even when immersed in the soft X-ray emission of the companion shell, as is often the case.

Indeed, the shell SNR IC 443, once though to be mostly thermal in the X-ray band (Petre et al. 1988; Asaoka \& Aschenbach 1994) has been discovered to emit hard X-ray emission by Wang et al. (1992). ASCA Gas Imaging Scintillator (GIS) observations by Keohane et al. (1997) discovered the localized character of the hard X-ray emission and its non-thermal nature. They concluded that most of the $2-10 \mathrm{keV}$ photons came from

Send offprint requests to: F. Bocchino,

e-mail: fbocchin@estec.esa.nl an isolated emitting feature and from the South East elongated ridge of hard emission. Preite-Martinez et al. (1999) and Bocchino \& Bykov (2000) reported a hard component detected with the Phoswich Detector System (PDS) on BeppoSAX and two compact X-ray sources corresponding to the ASCA sources detected with the BeppoSAX Medium-Energy Concentrator Spectrometer (MECS) (1SAX J0617.1+2221 and 1SAX J0618.0+2227). Very recently, 1SAX J0617.1+2221 has been observed by Chandra as reported by Olbert et al. (2001), who also show a VLA observation at 1.46, 4.86 and $8.46 \mathrm{GHz}$, and a polarization measurement. They argue that the hard radio spectral index, the amount of polarization and the overall X-ray and radio morphology strongly suggest that the source is a plerion nebula with a point source in it, whose characteristic cometary shape is due to supersonic motion of the neutron star. However, the limited counting statistics of the $10 \mathrm{ks}$ Chandra observation do not allow a detailed spectral study of the nebula, which is required to compare this new plerion with current theoretical models. Moreover, IC 443 is also a possible candidate for the CGRO EGRET $\gamma$-ray source $3 \mathrm{EG}$ J0617+2238 (Hartman et al. 1999) having a flux above $100 \mathrm{MeV}$ of $\sim 5 \times 10^{-7} \mathrm{ph} \mathrm{s}^{-1} \mathrm{~cm}^{-2}$ with a photon index of $2.01 \pm 0.06$. More detailed measurements of the nebula 
spectral properties are needed to study the relation between 3EG J0617+2238 and the nebula.

In this paper an XMM-Newton study of the recently discovered plerion nebula in IC 433 is presented. In particular, we use the large effective area of the EPIC instrument to address the synchrotron burn-off effect in the nebula, to resolve its structure and measure the flux, and to constrain the thermal radiation of the central object in the nebula.

\section{Observations}

IC 443 was observed as part of the Cal/PV phase of the $X M M-N e w t o n$ Observatory (Jansen et al. 2001). Here, we have used the two observations centered on $6^{\mathrm{h}} 17^{\mathrm{m}} 24^{\mathrm{s}} .3$ $+22^{\mathrm{d}} 26^{\mathrm{m}} 43^{\mathrm{s}}$ (J2000) performed on 2000 September 27. Data from the two MOS (Turner et al. 2001) cameras and the PN (Strueder et al. 2001) camera are used. The MOS and PN cameras are CCD arrays which collect X-ray photons between 0.1 and $15 \mathrm{keV}$ and have a field of view of $30^{\prime}$ diameter. The pixel size is $1.1^{\prime \prime}$ and $4.1^{\prime \prime}$ for MOS and PN, respectively, while the mirror Point Spread Function is $6^{\prime \prime}$ Full-width at half maximum. The data were acquired with the medium filter and in full image mode, and therefore the temporal resolution is $2.5 \mathrm{~s}$ and $73 \mathrm{~ms}$ for the MOS and PN, respectively. The poorer spatial resolution of the $\mathrm{PN}$ is compensated for by its greater sensitivity, on the average 20-30\% more than the combined MOS cameras.

The Standard Analysis System (SAS) software used (version 5.0.1, xmmsas-20001215) takes cares of most of the required events screening. However, we have further screened the data to eliminate some residual hot pixels and occasional background enhancement during intervals of intense incident flux of soft protons. In particular, we extracted the background lightcurve at energies $>10 \mathrm{keV}$ and identified time intervals of unusually high count rates (typically more than $1 \mathrm{~s}^{-1}$ ) and removed them from subsequent analysis. We merged the the event files of the subpointings before continuing with the analysis. The total screened and unscreened exposure times are 24 and $32 \mathrm{ks}$, respectively.

\section{Results}

\subsection{X-ray morphology}

We extracted PN images with a $4^{\prime \prime}$ pixel size and exposure images at the same resolution. We defined two energy bands in which to extract images, namely $0.5-2 \mathrm{keV}$ (hereafter the soft band) and 3-10 keV (hard band). Figure 1, top panel, shows the $0.5-2 \mathrm{keV}$ PN image of the South Eastern section of IC 443. Most of the emission in this band is of thermal origin, as established by Asaoka \& Aschenbach (1994) based on ROSAT data.

Figure 1, bottom panel, shows the $3-10 \mathrm{keV}$ PN image of the same field with a logarithmic color scaling and a heavier smoothing chosen in order to emphasize the faint diffuse emission. Most of the thermal emission associated with IC 443 is not present in this band, and therefore it is well suited to the study of any hard compact X-ray sources in the vicinity of this SNR. Indeed, this image shows several point sources, besides the plerion nebula itself. We see that the nebula is much more extended then reported by Olbert et al. (2001) and can be represented by an ellipse of $8^{\prime} \times 5^{\prime}$.

By fitting a two dimensional Gaussian to the image, we find that the location of the plerion nebula centroid is $6^{\mathrm{h}} 17^{\mathrm{m}} 05.5^{\mathrm{s}} 22^{\circ} 21^{\prime} 30^{\prime \prime} \quad(\mathrm{J} 2000$, therefore XMMU J061705.5+222130), with an estimated accuracy of $5^{\prime \prime}$, due to both statistical uncertainties (at $3 \sigma$ ) and systematic errors introduced by attitude reconstruction, and therefore in agreement with the position of the Chandra source CXOU J061705.3+222127. Figure 2 shows the combined MOS 3-10 keV image of the nebula core. The morphology of the faint rim matches somewhat that of the bright core, which may suggest a common origin, in agreement with the plerionic interpretation. Figure 2 also shows that the radio halo at $8.46 \mathrm{GHz}$ observed by Olbert et al. (2001) has somewhat different shape, and is smaller than the X-ray full extent of the nebula.

We also note that the source 1SAX J0618.0+2227 is resolved with XMM-Newton into two closeby sources (see Fig. 1). Moreover, there are other hard X-ray sources in the field of view. Some of these sources might be related to the SNR. The location of these sources is consistent with a scenario concerning the interaction of the SNR with molecular cloud, as proposed by Bocchino \& Bykov (2000).

\subsection{Spectral analysis}

\subsubsection{Contamination from soft thermal emission}

The spectral analysis of the plerion is rather complicated because of the presence of diffuse thermal emission on large spatial scales which is unassociated with the plerion nebula. Figure 1 shows that the plerion is partially superimposed on a large region of soft emission, running from the North East to the South West, which links the plerion region with the very bright soft region around $6^{\mathrm{h}} 17^{\mathrm{m}} 30^{\mathrm{s}} 22^{\circ} 30^{\prime}$.

In order to cope with these problems, we adopted the following procedure. First, we identified a "background area" (Bkg in Fig. 1) which is outside the thermal emission of IC 443. This background have been corrected for vignetting and area effects prior to subtraction of the source spectra. The background correctly includes emission from G189.6+3.3, the "companion" SNR identified by Asaoka \& Aschenbach (1994), which encompasses all the field of view of the XMM-Newton observation. Then, we defined a sample of the thermal region in which the plerion is partially immersed (Th2 in Fig. 1). Unfortunately, the Th2 region cannot be located outside the plerion nebula, because the spectral properties may be different in a region so far from the nebula. The thermal region was fitted with the two-temperature optically thin plasma model of Mewe et al. (1985) and Liedahl et al. (1995) with interstellar 


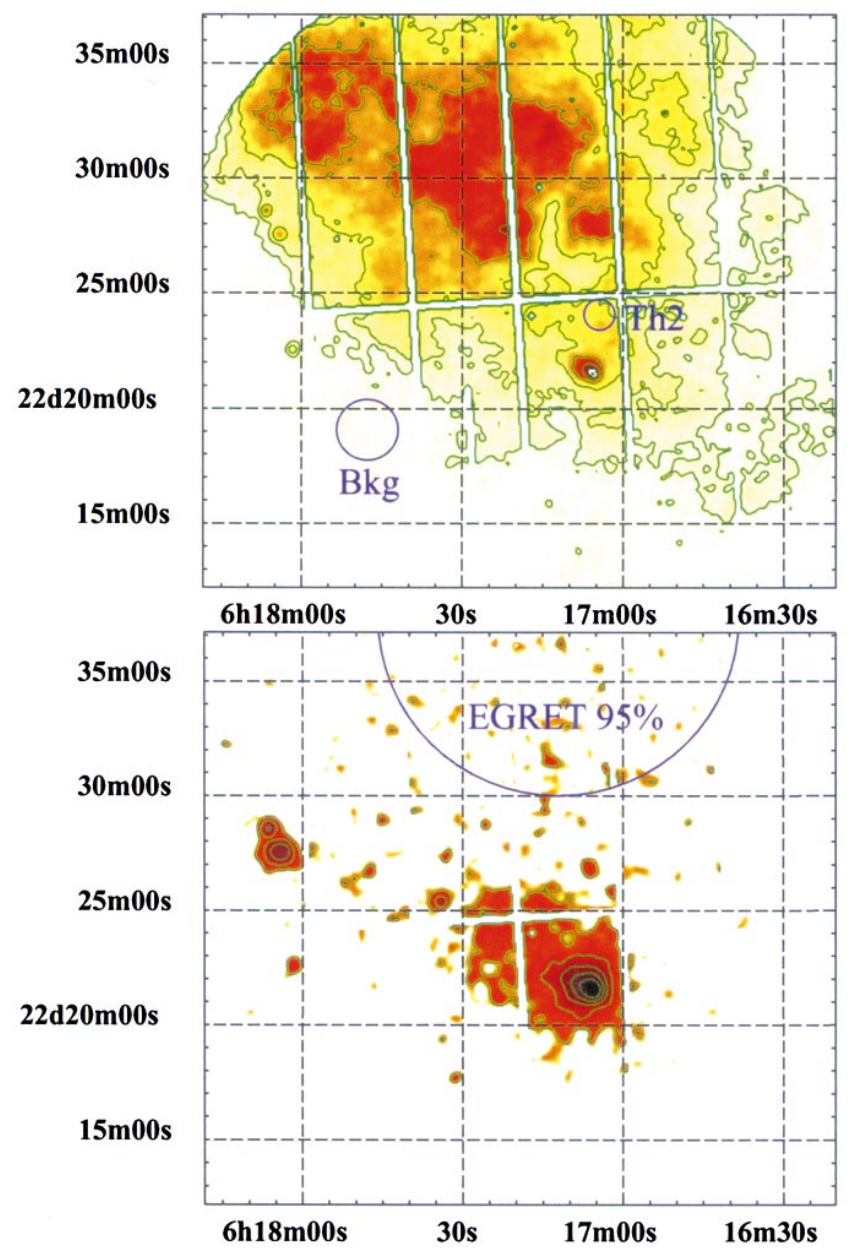

Fig. 1. $4^{\prime \prime}$ pixel PN images of the IC 443 plerion. Top: soft energy band $(0.5-2 \mathrm{keV})$, linear colorscale, logarithmic contours between $1 / 16$ and the peak value, 30 count pixel ${ }^{-1}$, each contour is a factor of two from the previous and smoothed with a 1.5 pixel $\sigma$ Gaussian. Bottom: hard energy band $(3-10 \mathrm{keV})$, $\log$ colorscale, same log contours spacing but between $1 / 32$ and the peak at 12.5 count pixel ${ }^{-1}$, smoothed with a 3 pixel $\sigma$ Gaussian. The $95 \%$ confidence EGRET error circle for 3EG $\mathrm{J} 0617+2238$ is also shown.

absorption of Morrison \& McCammon (1983), using the appropriate PN response matrix for off-axis sources. An additional power-law component was used to model the non-thermal emission of the plerion falling inside the Th2 extraction region. The above $2 \mathrm{~T}$ model has been successfully used by Asaoka \& Aschenbach (1994) to describe the ROSAT data of this part of IC 443. A proper study of the remnant thermal emission including more appropriate models (e.g., Non-Equilibrium of Ionization) is beyond the scope of our present work.

The results obtained from the thermal regions show that the two temperature thermal model used by Asaoka \& Aschenbach (1994) provides a good description of the data with best-fit parameters in agreement with the findings of ROSAT $\left(k T_{1}=0.18_{-0.03}^{+0.10}, k T_{2}=0.90_{-0.05}^{+0.10}, N_{\mathrm{H}}=\right.$ $8.0 \pm 1.5 \times 10^{21}, \chi^{2} /$ dof $\left.=71 / 66\right)$.

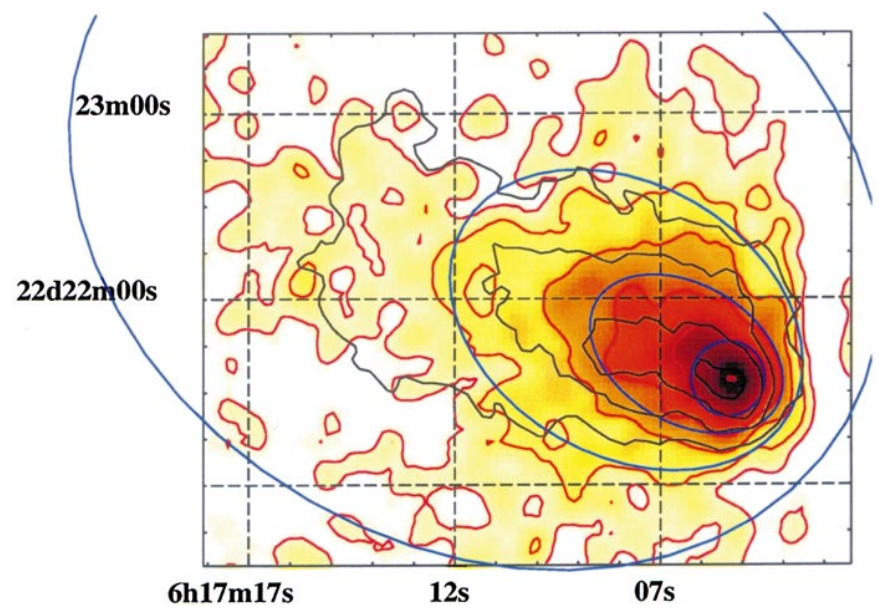

Fig. 2. MOS1+2 3-10 keV image closeup of the core of the nebula, smoothed with a Gaussian of $4^{\prime \prime} \sigma$. Black contours are drawn from Olbert et al. 2001, at $8.46 \mathrm{GHz}$, in steps of $1 \mathrm{mJy}$ beam $^{-1}$. The elliptical regions used in spectral analysis are also displayed, as well as logarithmic X-ray contours between 1/32 and the peak value ( 8 count pixel ${ }^{-1}$ ), each one a factor of two from the previous.

\subsubsection{The central compact source}

We analyzed the spectrum of the compact source XMMU J061705.5+222130, extracting its spectrum from a $6^{\prime \prime}$ radius region centered on the source centroid. In order to take into account the residual thermal emission in the fittings, we used a power-law model together with the $2 \mathrm{~T}$ model used in the Th2 region to fit the data. The absorption, temperatures and normalization ratio of the two thermal component were kept fixed to the values found in Th2. The best-fit results are reported in Table 1. Since the best-fit normalization of the thermal component is a factor of 2 higher than that expected by scaling the value found in Th2, we have tested for the presence of an additional black-body component in the spectrum of this central part of the nebula, by adding this component to the power-law instead of the $2 \mathrm{~T}$ component. There is no a significant improvement in $\chi^{2}$, but a best-fit is found with $k T=0.13 \mathrm{keV}$ and a neutron star (NS) radius of $3.3 \mathrm{~km}$. The corresponding values of the black-body temperature and radius as observed at infinite distance allowed by our data are shown in Fig. 3. At the temperature derived by Olbert et al. (2001) with Chandra, $k T=0.7 \mathrm{keV}$, the upper-limit to the $1-5 \mathrm{keV}$ unabsorbed flux of the $\mathrm{BB}$ component is $4.4 \times 10^{-13} \mathrm{erg} \mathrm{cm}^{-2} \mathrm{~s}^{-1}$, (consistent with the Chandra detection of a flux of $\left.2 \times 10^{-13} \mathrm{erg} \mathrm{cm}^{-2} \mathrm{~s}^{-1}\right)$, but the high temperature and very low emitting area (even for heated polar cap models) points against this region of the parameter space.

We have also investigated the presence of periodic signal by performing an FFT of the PN events collected in the NS source region, i.e. a circular region centered on the centroid with a $6^{\prime \prime}$ radius. We did not find a periodic signal at the $99 \%$ confidence level in the $10^{-4}-6.5 \mathrm{~Hz}$ range, with an upper limit, at the same confidence level, of $16.8 \%$ of sinusoidal pulsated fraction. 


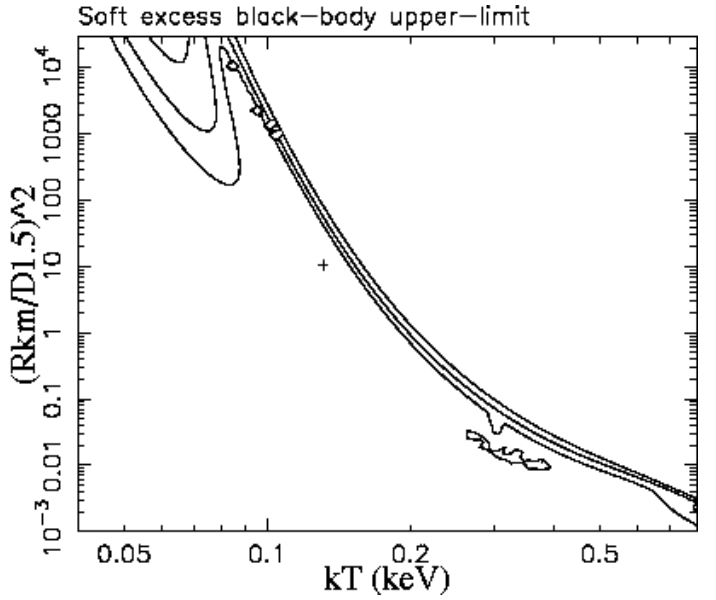

Fig. 3. Upper limit to the blackbody component in the spectral fits of XMMU J061705.5+222130. We present $68 \%, 90 \%$ and $99 \%$ confidence level $\chi^{2}$ contours for 4 interesting parameters versus the blackbody temperature and normalization (in units of $\left(R_{\mathrm{km}} / D_{1.5}\right)^{2}$, where $R_{\mathrm{km}}$ is the blackbody radius in $\mathrm{km}$ and $D_{1.5}$ is the distance in units of $1.5 \mathrm{kpc}$. The best-fit values are marked with a cross.

\subsubsection{The diffuse nebula}

In order to test the plerionic interpretation, we have tried to detect the softening of the spectrum toward the outer regions of the nebula, a well known effect which has been observed in other plerions (e.g. 3C 58, Torii et al. 2000; Bocchino et al. 2001; G21.5-0.9 by Slane et al. 2000; Warwick et al. 2001) and which is caused by the short lifetime of high energy electrons compared with those with lower energies. Given the peculiar shape of this nebula, we extracted spectra from four elliptical annuli, shown in Fig. 2, and fit them separately. We chose the ellipse sizes in order to minimize the point spread function (PSF) contamination effects. For instance, the first region is a $12^{\prime \prime}$ radius circle, in which $\sim 75 \%$ of the PSF integral is included.

Because of the contamination by thermal emission, we have followed the same approach used in fitting the NS region, i.e. a fixed parameter $2 \mathrm{~T}$ model and a power-law. Whilst this procedure does not take into account the uncertainties associated in the Th2 fit (which however are not large), it provides an accurate way of not rejecting useful data, thus reducing the overall uncertainties in the spectral parameter of the nebula. For comparison, we also show the results obtained with fits to the hard band spectrum $(E>3 \mathrm{keV})$ and a single power-law.

The results of the spectral analysis are shown in Table 1 and the spectrum of the central core is shown in Fig. 4. All the fits provide a good description of the data. In Fig. 5 we present the spectral softening in the nebula going from the inner to the outer regions, as well as the softening observed in other plerions, for comparison. Letting the absorption value vary does not yield a
Table 1. Summary of PN spectral fitting results.

\begin{tabular}{lcccc}
\hline Name & $\gamma^{a}$ & $\gamma^{b}$ & $\begin{array}{c}\mathrm{flux}^{c} \times 10^{-13} \\
\mathrm{erg} \mathrm{cm}^{-2} \mathrm{~s}^{-1}\end{array}$ & $\chi^{2} /$ dof \\
\hline NS & - & $1.63_{-0.17}^{+0.11}$ & $6.8 \pm 1.7$ & $27 / 24$ \\
PlCore & $1.71_{-0.23}^{+0.44}$ & $1.63_{-0.10}^{+0.11}$ & $6.3 \pm 0.8$ & $40 / 48$ \\
Pl1 & $1.95_{-0.18}^{+0.35}$ & $1.81_{-0.06}^{+0.08}$ & $12.8 \pm 1.1$ & $119 / 108$ \\
Pl2 & $1.88_{-0.16}^{+0.31}$ & $1.88_{-0.06}^{+0.07}$ & $13.8 \pm 1.2$ & $149 / 149$ \\
Pl3 & $2.70_{-0.26}^{+0.31}$ & $2.30_{-0.09}^{+0.09}$ & $17.2 \pm 1.7$ & $451 / 435$ \\
\hline
\end{tabular}

${ }^{a}$ Fit to power-law only, $3-10 \mathrm{keV}, N_{\mathrm{H}}=8 \times 10^{21} \mathrm{~cm}^{-2}$.

${ }^{b}$ Fit to power-law + fixed parameters $2 \mathrm{~T}$ components $\left(N_{\mathrm{H}}=\right.$ $8 \times 10^{21}, k T_{1}=0.2, k T_{2}=0.9$, norm $_{1} /$ norm $_{2}=10$. Full bandwidth $(0.5-10 \mathrm{keV})$.

${ }^{c}$ Power-law unabsorbed flux in the $2-10 \mathrm{keV}$ band.
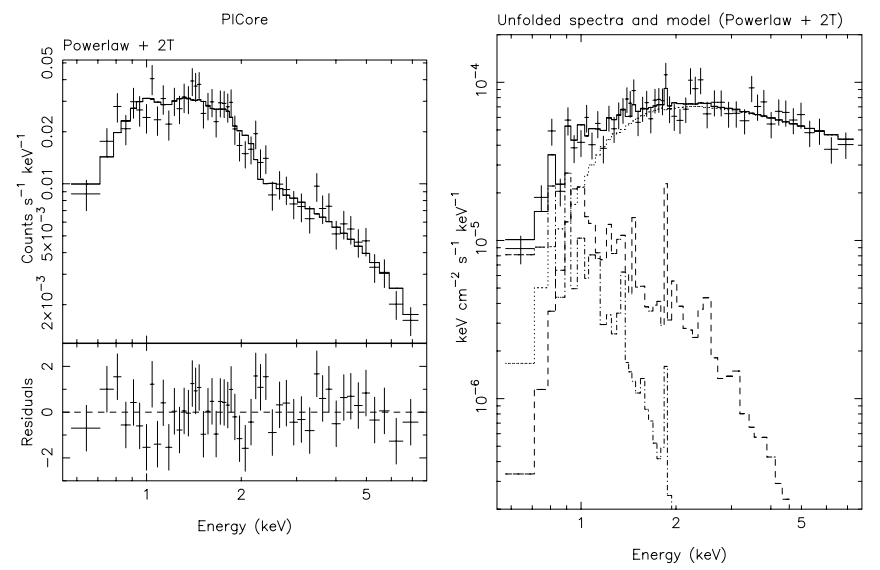

Fig. 4. Folded and unfolded PN spectrum of the central region of the plerion (within 12" from XMMU J061705.5+222130). The best-fit model (a power-law and two component thermal plasma with parameter derived from the Th2 region) is also shown. In the unfolded panel, the contribution of the thermal components (dashed and dot-dashed) and the power-law (dotted) are shown.

better fit, therefore we argue that there is no evidence for any absorption variation across the plerion nebula.

\section{Discussion}

The XMM-Newton observations allow us to resolve the structure of the hard X-ray source XMMU J061705.5+222130. The observed power-law index steepening (Fig. 5) is consistent with the synchrotron burn-off effect (Kennel \& Coroniti 1984) and remarkably similar to the effect observed in other plerions, thus confirming the pulsar wind nebula interpretation suggested by Olbert et al. (2001). Note that the frequency index steepening is consistent with the standard value of 0.5 from the one-zone model by Chevalier (2000), and that the hard spectral index of the core suggests a low X-ray efficiency, similar to the Vela-X nebula and CTB 80 as reported by Chevalier (2000). Following the one-zone Chevalier (2000), away from cooling regime the $L_{\mathrm{X}} / \dot{E}$ ratio is dependent on the fraction $\epsilon_{\mathrm{B}}$ of energy 


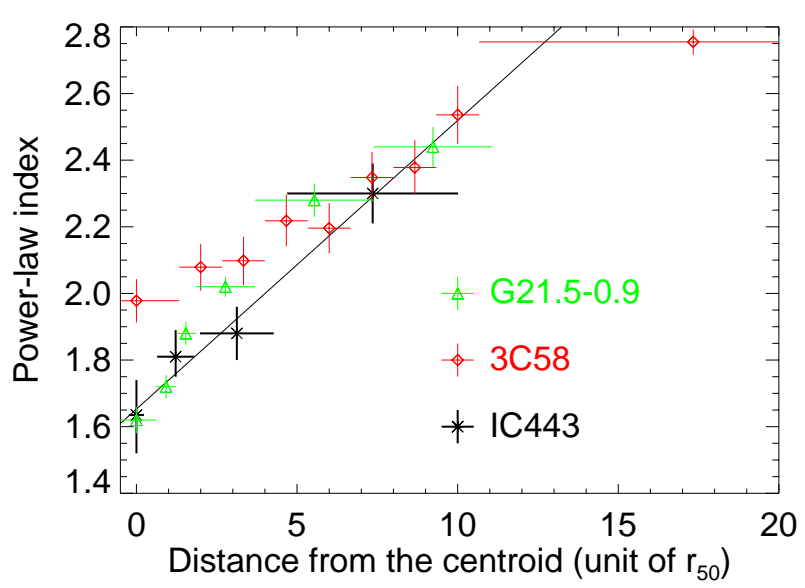

Fig. 5. $\gamma-r$ relation in the IC 443 plerion nebula (this paper), 3C 58 (Bocchino et al. 2001) and G21.5-0.9 (Warwick et al. 2001). The $X$-axis shows the weighted mean distance of the pixels of a given region from the centroid of the nebula, expressed in unit of $r_{50}$, the radius at which the plerion surface brightness drop by a factor of $2\left(12^{\prime \prime}, 6^{\prime \prime}\right.$ and $13^{\prime \prime}$ for IC 443 , $3 \mathrm{C} 58$ and G21.5-0.9, respectively). Using the $r_{50}$ unit gives a measure independent on the distance to the nebulae, while $r_{50}$ can be easily converted into angular or absolute distances using the SNR distances and above angular measures of $r_{50}$. The positional error bar are the standard deviation of the distances. A fit with a linear relation for IC 443 only is also plotted.

density of the emitting region going into magnetic fields $\left(L_{\mathrm{X}} / \dot{E} \propto \epsilon_{\mathrm{B}}^{0.83}\right)$, and this quantity is rather uncertain. However, assuming $L_{\mathrm{X}} / \dot{E}=0.002$, like in the bow-shock nebula CTB 80, and using the sum of the flux in all the elliptical regions $\left(2.6 \times 10^{33} \mathrm{erg} \mathrm{s}^{-1}\right.$ in the $0.2-4 \mathrm{keV}$ band at $1.5 \mathrm{kpc})$, we obtain $\dot{E}=1.3 \times 10^{36} \mathrm{erg} \mathrm{s}^{-1}$ $\left(0.8 \times 10^{36} \mathrm{erg} \mathrm{s}^{-1}\right.$ with the empirical relation of Seward \& Wang 1988), similar to the value adopted by Olbert et al. (2001).

Another important characteristic of this plerion nebula is that its X-ray extent appears larger then its radio extension, an effect also observed in G21.5-0.9 (Warwick et al. 2001) and 3C 58 (Bocchino et al. 2001). This is unexpected if both X-ray and radio are just the synchrotron emission of the nebula. However, the inverse Compton and nonthermal bremsstrahlung components of the X-ray emission, as well as other thermal components related to a possible shell, may become important in the outer halo of the nebula. Unfortunately, there is no way to verify if, apart from the contamination of the IC 443 thermal emission, the spectrum of the outer nebula is purely non-thermal (like in G21.5-0.9, Warwick et al. 2001), or contains an additional thermal component due to the expansion of the nebula into the ejecta (as in 3C 58, Bocchino et al. 2001). On the other hand, such a small radio nebula may be the result of a low sensitivity of the VLA observations reported by Olbert et al. (2001). The X-ray to radio luminosity ratio, defined for instance as in Woltjer et al. (1997), is only a factor of 2 less then the Crab's and 100 more than 3C 58. But if deeper radio observations reveal a nebula comparable with, or larger then, the X-ray nebula the real value of the ratio may be reduced.

By extrapolating back the sum of the spectra of the different nebula regions in the radio regime, and comparing them with the radio spectrum reported by Olbert et al. (2001), we have found a spectral break at $100 \mathrm{GHz}$, instead of $10000 \mathrm{GHz}$ as reported by Olbert et al. (2001). The difference is due to the contribution of the hard inner nebula regions, which were not properly modeled using ASCA data, on which the Olbert et al. (2001) estimation relies upon. Such a low frequency break is also found in 3C 58 and G21.5-0.9, as well as in other examples of other "non Crab-like" plerion reported by Woltjer et al. (1997).

It should be noted, that there are some differences between the IC 443 plerion and 3C 58 (and G21.5-0.9). In particular, the spectral index steepening in $3 \mathrm{C} 58$ and G21.5-0.9 is higher than 0.5, the value predicted by the one-zone model of Chevalier (2000). Another key difference is the central source in the center, which is very prominent in case of the IC 443 plerion. However, on the basis of the published Chandra data on G21.5-0.9 (Slane et al. 2000), the presence of central compact sources in G21.5-0.9 cannot be excluded.

As for the NS, a better interpretation of the its temperature constraint requires a careful analysis of the NS atmosphere effect (e.g., Pavlov et al. 1995) and general relativitistic corrections. The effect of the hydrogen atmosphere could reduce the effective temperature typically by a factor 1.3 to 2 and thus could make the effective NS radius consistent with the standard NS radius of $\sim 10 \mathrm{~km}$. It is worth noting that the NS temperature of $k T \sim 0.1 \mathrm{keV}$ is nicely consistent with the IC 443 age of 30000 years (Chevalier 1999), if the standard NS cooling curve of superfluid NS is used (e.g., Yakovlev et al. 1999, - see their Fig. 7), but a lower age cannot be excluded. The discrepancy between our best guess for the NS surface temperature and the temperature derived by Olbert et al. (2001) by fitting the point source CXOU J061705.3+222127 may be due to the low value of $N_{\mathrm{H}}$ assumed by them $\left(1.3 \times 10^{21} \mathrm{~cm}^{-2}\right)$. We verified that this $N_{\mathrm{H}}$ value is rejected at $99 \%$ confidence level by all our fits. A deeper Chandra observation will better constrain the physical properties of the compact source.

If the hard power-law spectrum of the nebula core region is extrapolated up to $\mathrm{GeV}$ regime, it would provide a flux of $2.0(0.4-15.2) \times 10^{-7} \mathrm{ph} \mathrm{cm}^{-2} \mathrm{~s}^{-1}$, which is consistent with the EGRET flux of 3EG J0617+2238 $\left(5.0 \pm 0.4 \times 10^{-7} \mathrm{ph} \mathrm{cm}^{-2} \mathrm{~s}^{-1}\right)$. The nebula is placed outside the $95 \%$ uncertainty circle of the $3 \mathrm{EG} \mathrm{J} 0617+2238$, but the systematic uncertainties of the EGRET circle allows us to speculate about possible association of the two sources. If, however, the $\mathrm{GeV}$ regime EGRET photons indeed originate from synchrotron emission of relativistic electrons, this would seriously constrain the nebula model of Kennel \& Coroniti (1984) where $\mathrm{e}^{ \pm}$are accelerated by a relativistic wind termination shock and then radiate in the downstream region. The maximum photon energy $\varepsilon$ (measured in $\mathrm{GeV}$ ) produced by an accelerated 
electron of maximal Lorentz factor $\Gamma_{\max }$ in the magnetic field of the emitting region $B_{\mathrm{e}}$ (measured in $\mathrm{G}$ ) can be obtained from $8.6 \times 10^{16} \varepsilon \approx B_{\mathrm{e}} \Gamma_{\max }^{2} \mathrm{G}$. The $\mathrm{e}^{ \pm}$synchrotron loss time, $t_{\mathrm{s}} \approx 5 \times 10^{8} B_{\mathrm{a}}^{-2} \Gamma^{-1} \mathrm{~s}$, must exceed the $\mathrm{e}^{ \pm}$acceleration time by relativistic shock, $t_{\mathrm{a}}$ (where $B_{\mathrm{a}}$ is the magnetic field in the acceleration region). Since $t_{\mathrm{a}}$ cannot be less then the particle gyroperiod, one may obtain $B_{\mathrm{a}} \Gamma_{\max }^{2} \ll 10^{16} \mathrm{G}$. Thus, the magnetic field amplification in the shock downstream region $B_{\mathrm{e}} \gg 8 B_{\mathrm{a}} \varepsilon$ is required. The amplification effect can be studied with high spatial resolution X-ray imaging. The EGRET luminosity of $\sim 10^{35} \mathrm{erg} \mathrm{s}^{-1}$ would not be a negligible fraction of the spin-down power estimated from the Seward \& Wang (1988) relation. An alternative explanation for the EGRET emission is the relativistic bremsstrahlung of radio emitting electrons accelerated by the supernova radiative shock (Bykov et al. 2000), and future INTEGRAL and GLAST observations will resolve that alternative, as well as providing an accurate position for 3EG J0617+2238.

\section{Summary and conclusions}

We report $X M M-N$ ewton $\mathrm{Cal} / \mathrm{PV}$ observations of the hard X-ray nebula inside the supernova remnant IC 443, recently discovered by Chandra (Olbert et al. 2001). The longer exposure time and the high XMM-Newton effective area have allowed us to trace and to study the diffuse emission of the nebula far beyond the extension reported by Olbert et al. (2001). We confirm the plerionic nature of the nebula, as suggested by Olbert et al. (2001), by virtue of the observation of the synchrotron burn-off, which is in agreement with plerion model expectations. The relatively hard power-law index of the core, the flat radio spectrum, the low frequency break, and the X-ray extension much larger then the radio extension at $8.46 \mathrm{GHz}$ make this plerion more similar to $3 \mathrm{C} 58$ and G21.5-0.9 (two well known examples of "non Crab-like" or "second kind" plerions, Woltjer et al. 1997), rather than to the Crab nebula. Our data allows us to place only upper-limit on the presence of a thermal compact X-ray source in the nebula core. However, we argue that its temperature is likely be $\sim 0.1 \mathrm{keV}$, significantly lower that the temperature suggested by Olbert et al. (2001). We also argue that, if the nebula is the counterpart of the EGRET source 3EG J0617+2238, as not excluded by the extrapolation of its X-ray spectrum, a strong constraint on magnetic field amplification in the nebula may be posed.

Acknowledgements. F. Bocchino acknowledges an ESA Research Fellowship. The work of A.M.B was supported by the INTAS-ESA 99-1627 grant. We thank A. N. Parmar and the anonymous referee for their useful comments on the manuscript. We also thank the ESTEC XMM-Newton staff for their suggestions about data analysis.

\section{References}

Asaoka, I., \& Aschenbach, B. 1994, A\&A, 284, 573

Bocchino, F., \& Bykov, A. M. 2000, A\&A, 362, L29

Bocchino, F., Warwick, R. S., Marty, P., et al. 2001, A\&A, 369, 1078

Bykov, A. M., Chevalier, R. A., Ellison, D. C., \& Uvarov, Y. A. 2000, ApJ, 538, 203

Chevalier, R. A. 1999, ApJ, 511, 798

Chevalier, R. A. 2000, ApJ, 539, L45

Hartman, R. C., Bertsch, D. L., Bloom, S. D., et al. 1999, ApJS, 123, 79

Helfand, D. J., Gotthelf, E. V., \& Halpern, J. P. 2001 [astro-ph/0007310]

Jansen, F., Lumb, D., Altieri, B., et al. 2001, A\&A, 365, L1

Kennel, C. F., \& Coroniti, F. V. 1984, ApJ, 283, 694

Keohane, J. W., Petre, R., Gotthelf, E. V., Ozaki, M., \& Koyama, K. 1997, ApJ, 484, 350

Liedahl, D. A., Osterheld, A. L., \& Goldstein, W. H. 1995, ApJ, 438, L115

Mewe, R., Gronenschild, E. H. B. M., \& van den Oord, G. H. J. 1985, A\&AS, 62, 197

Morrison, R., \& McCammon, D. 1983, ApJ, 270, 119

Olbert, C., Clearfield, R. C., Williams, N., Keohane, J., \& Frail, D. A. 2001, ApJ, 554, L205

Pavlov, G. G., Shibanov, Y. A., Zavlin, V. E., \& Meyer, R. D. 1995, The Lives of the Neutron Stars, ed. M. A. Alpar, 71

Pavlov, G. G., Zavlin, V. E., Sanwal, D., Burwitz, V., \& Garmire, G. P. 2001, ApJ, 552, L129

Petre, R., Szymkowiak, A. E., Seward, F. D., \& Willingale, R. 1988, ApJ, 335, 215

Preite-Martinez, A., Feroci, M., Strom, R. G., \& Mineo, T. 1999, AIP Conf. Proc, 510, 73

Seward, F. D., \& Wang, Z. 1988, ApJ, 332, 199

Slane, P., Chen, Y., Schulz, N. S., et al. 2000, ApJ, 533, L29

Strueder, L., Briel, U., Dennerl, K., et al. 2001, A\&A, 365, L18

Torii, K., Slane, P. O., Kinugasa, K., Hashimotodani, K., \& Tsunemi, H. 2000, PASJ, 52, 875

Turner, M., Abbey, A., Arnaud, M., et al. 2001, A\&A, 365, L27

Wang, Z. R., Asaoka, I., Hayakawa, S., \& Koyama, K. 1992, PASJ, 44, 303

Warwick, R. S., Bernard, J., Bocchino, F., et al. 2001, A\&A, 365, L248

Woltjer, L., Salvati, M., Pacini, F., \& Bandiera, R. 1997, A\&A, 325,295

Yakovlev, D. G., Kaminker, A. D., \& Levenfish, K. P. 1999, A\&A, 343, 650 